\title{
On almost sure limiting behavior of a dependent random sequence
}

\section{Ai-hua Fan* and Zhong-zhi Wang}

"Correspondence: fah@ahut.edu.cn School of Mathematics \& Physics, AnHui University of Technology, Ma'anshan, 243002, People's

Republic of China

\begin{abstract}
We study some sufficient conditions for the almost certain convergence of averages of arbitrarily dependent random variables by certain summability methods. As corollaries, we generalized some known results.
\end{abstract}

MSC: $60 \mathrm{~F} 15$

Keywords: dependent random variable; summability; dominated random sequence

\section{Introduction}

In reference [1], Chow and Teicher gave a limit theorem of almost certain summability of i.i.d. random variables as follows.

Theorem (Chow et al., 1971) Let $a(x), x>0$ be a positive non-increasing function and $a_{n}=a(n), A_{n}=\sum_{k=1}^{n} a_{k}, b_{n}=A_{n} / a_{n}$, where

(1) $A_{n} \rightarrow \infty$;

(2) $0<\liminf _{n} \frac{b_{n}}{n} a\left(\log b_{n}\right) \leq \lim \sup _{n} \frac{b_{n}}{n} a\left(\log b_{n}\right)<\infty$;

(3) $x a\left(\log ^{+} x\right)$ is non-decreasing for $x>0$, then i.i.d. $\left\{X, X_{n}\right\}$ are $a_{n}$ summable, i.e.,

$$
T_{n}=A_{n}^{-1} \sum_{k=1}^{n} a_{k} X_{k}-C_{n} \rightarrow 0 \quad \text { a.c. }
$$

for some choice of centering constants $C_{n}$, if and only if

$$
E|X| a\left(\log ^{+}|X|\right)<\infty
$$

Motivated by Chow and Teicher's idea, in this paper we consider the problem of arbitrarily dependent random variables and their limiting behavior from a new perspective.

Throughout this paper, let $\mathbb{N}$ denote the set of positive integers, $\left\{X, X_{n}, \mathcal{F}_{n}, n \in \mathbb{N}\right\}$ be a stochastic sequence defined on the probability space $(\Omega, \mathcal{F}, \mathbb{P})$, i.e., the sequence of $\sigma$ fields $\left\{\mathcal{F}_{n}, n \in \mathbb{N}\right\}$ in $\mathcal{F}$ is increasing in $n$, and $\left\{\mathcal{F}_{n}\right\}$ are adapted to random variables $\left\{X_{n}\right\}$, $\mathcal{F}_{0}$ denotes the trivial $\sigma$ field $\{\Phi, \Omega\}$ and $\mathbf{1}_{[\cdot]}$ the indicator function.

We begin by introducing some terminology and lemmas.

Definition 1 (Adler et al., 1987 [2]) Let $\left\{X_{n}, n \in \mathbb{N}\right\}$ be a sequence of random variables, and it is said to be stochastically dominated by a random variable $X$ (we write $\left\{X_{n}, n \in \mathbb{N}\right\} \prec X$ ) 
if there exists a constant $C>0$, for almost every $\omega \in \Omega$, such that

$$
\sup _{n \in \mathbb{N}} \mathbb{P}\left\{\left|X_{n}\right|>t\right\} \leq C \mathbb{P}\{|X|>t\} \quad \text { for all } t>0
$$

Lemma 1 (Chow et al., 1978 [3]) Let $\left\{X_{n}, \mathcal{F}_{n}, n \in \mathbb{N}\right\}$ be an $L_{p}(1 \leq p \leq 2)$ martingale difference sequence, if $\sum_{n=1}^{\infty} E\left(\left|X_{n}\right|^{p} \mid \mathcal{F}_{n-1}\right)<\infty$, then $\sum_{n=1}^{\infty} X_{n}$ a.c. converges.

Lemma 2 Let $\left\{X, X_{n}, n \in \mathbb{N}\right\}$ be a sequence of random variables. If $\left\{X_{n}\right\} \prec X$, then for all $t>0$,

$$
\mathbb{E}\left|X_{n}\right|^{2} \mathbf{1}_{\left[\left|X_{n}\right| \leq t\right]} \leq C\left[t^{2} \mathbb{P}(|X|>t)+\mathbb{E} X^{2} \mathbf{1}_{[|X| \leq t]}\right]
$$

Proof By the integral equality

$$
2 \int_{0}^{t} s \mathbb{P}\left(\left|X_{n}\right|>s\right) d s=t^{2} \mathbb{P}\left(\left|X_{n}\right|>t\right)+\mathbb{E}\left|X_{n}\right|^{2} \mathbf{1}_{\left[\left|X_{n}\right| \leq t\right]},
$$

it follows that

$$
\begin{aligned}
& \mathbb{E}\left|X_{n}\right|^{2} \mathbf{1}_{\left[\left|X_{n}\right| \leq t\right]} \leq 2 \int_{0}^{t} s \mathbb{P}\left(\left|X_{n}\right|>s\right) d s \\
& \quad \leq 2 C \int_{0}^{t} s \mathbb{P}(|X|>s) d s=C\left[t^{2} \mathbb{P}(|X|>t)+\mathbb{E} X^{2} \mathbf{1}_{[|X| \leq t]}\right] .
\end{aligned}
$$

\section{Strong law of large numbers}

In this section, we always assume that $a(x), x>0$ is a positive non-increasing function and $a_{n}=a(n), A_{n}=\sum_{k=1}^{n} a_{k}, b_{n}=A_{n} / a_{n}$, where

(1) $A_{n} \rightarrow \infty$;

(2) $0<\liminf _{n} \frac{b_{n}}{n} a\left(\log b_{n}\right) \leq \lim \sup _{n} \frac{b_{n}}{n} a\left(\log b_{n}\right)<\infty$;

(3) $x a\left(\log ^{+} x\right)$ is non-decreasing for $x>0$.

Theorem 1 Let $\left\{X, X_{n}\right\}$ be a sequence of random variables with $\left\{X_{n}\right\} \prec X$. If $E|X| a\left(\log ^{+}|X|\right)<\infty$, then

$$
\lim _{n} \frac{1}{A_{n}} \sum_{k=1}^{n} a_{k}\left[X_{k}-E\left(X_{k} \mathbf{1}_{\left[\left|X_{k}\right| \leq b_{k}\right]} \mid \mathcal{F}_{k-1}\right)\right]=0, \quad \text { a.c. }
$$

Proof To prove (2.1) by applying the Kronecker lemma, it suffices to show that

$$
\text { the series } \sum_{n=1}^{\infty} \frac{X_{n}-E\left(X_{n} \mathbf{1}_{\left[\left|X_{n}\right| \leq b_{n}\right]} \mid \mathcal{F}_{n-1}\right)}{b_{n}} \quad \text { converges a.c. }
$$

Since $0<a(x) \downarrow$, (1) guarantees that $b_{n} \uparrow \infty$. Choose $m_{0}$ such that $n \geq m_{0}$ implies

$$
\alpha n \leq b_{n} a\left(\log b_{n}\right) \leq \beta n
$$

whence $b_{n} \geq \alpha n\left[a\left(\log b_{m}\right)\right]^{-1}$ for $n \geq m \geq m_{0}$ entailing

$$
\sum_{k=m}^{\infty} b_{k}^{-2} \leq \frac{a^{2}\left(\log b_{m}\right)}{\alpha^{2} m}
$$


Put $Y_{n}=X_{n} \mathbf{1}_{\left[\left|X_{n}\right| \leq b_{n}\right]}, Z_{n}=X_{n} \mathbf{1}_{\left[\left|X_{n}\right|>b_{n}\right]}$, obviously, $X_{n}=Y_{n}+Z_{n}, n \in \mathbb{N}$. Note that $\left\{X_{n}\right\} \prec X$ and the condition $E|X| a\left(\log ^{+}|X|\right)<\infty$, we have

$$
\begin{aligned}
& \sum_{n=1}^{\infty} \mathbb{P}\left(\left|X_{n}\right|>b_{n}\right) \\
& \quad=\sum_{n=1}^{m_{0}-1} \mathbb{P}\left(\left|X_{n}\right|>b_{n}\right)+\sum_{n=m_{0}}^{\infty} \mathbb{P}\left(\left|X_{n}\right|>b_{n}\right) \\
& \leq m_{0}-1+C \sum_{n=m_{0}}^{\infty} \mathbb{P}\left(|X|>b_{n}\right) \\
& \leq m_{0}-1+C \sum_{n=m_{0}}^{\infty} \mathbb{P}\left(|X| a(\log |X|) \geq b_{n} a\left(\log b_{n}\right)\right) \\
& \leq m_{0}-1+C \sum_{n=m_{0}}^{\infty} \mathbb{P}(|X| a(\log |X|) \geq \alpha n)<\infty,
\end{aligned}
$$

which shows

$$
\mathbb{P}\left(X_{n} \neq Z_{n}, \text { i.o. }\right)=0 \text {. }
$$

Let $W_{n}=\frac{Y_{n}}{b_{n}}-E\left(\frac{Y_{n}}{b_{n}} \mid \mathcal{F}_{n-1}\right)$, then $\left(W_{n}, \mathcal{F}_{n}, n \in \mathbb{N}\right)$ is a martingale difference sequence.

Since

$$
\begin{aligned}
& \sum_{k=1}^{\infty} E \frac{Y_{k}^{2}}{b_{k}^{2}}=\sum_{k=1}^{\infty} \frac{E X_{k}^{2} \mathbf{1}_{\left[\left|X_{k}\right| \leq b_{k}\right]}}{b_{k}^{2}} \\
& \leq C \sum_{k=1}^{\infty}\left[E \mathbf{1}_{\left[|X|>b_{k}\right]}+\frac{E X^{2} \mathbf{1}_{\left[|X| \leq b_{k}\right]}}{b_{k}^{2}}\right] \quad(\text { by Lemma 2) } \\
& =C \sum_{k=1}^{\infty} E \mathbf{1}_{\left[|X|>b_{k}\right]}+C\left(\sum_{k=1}^{m_{0}-1}+\sum_{k=m_{0}}^{\infty}\right) \frac{E X^{2} \mathbf{1}_{\left[|X| \leq b_{k}\right]}}{b_{k}^{2}} \\
& \leq C \sum_{k=1}^{\infty} \mathbb{P}\left(|X|>b_{k}\right)+C\left(m_{0}-1\right)+C \sum_{k=m_{0}}^{\infty} b_{k}^{-2}\left(\int_{\left[\left|X_{k}\right| \leq b_{m_{0}-1}\right]} X^{2}+\sum_{i=m_{0}}^{k} \int_{\left[b_{i-1}<|X| \leq b_{i}\right]} X^{2}\right) \\
& \leq \mathcal{O}(1)+C \sum_{i=m_{0}}^{\infty} \sum_{k=i}^{\infty} b_{k}^{-2} \int_{\left[b_{i-1}<|X| \leq b_{i}\right]} X^{2} \quad(\text { by }(2.4)) \\
& \leq \mathcal{O}(1)+\alpha^{-2} C \sum_{i=m_{0}}^{\infty} i^{-1} a^{2}\left(\log b_{i}\right) \int_{\left[b_{i-1}<|X| \leq b_{i}\right]} X^{2} \quad \text { (by (2.3)) } \\
& \leq \mathcal{O}(1)+\alpha^{-2} \beta C \sum_{i=m_{0}}^{\infty} a\left(\log b_{i}\right) \int_{\left[b_{i-1}<|X| \leq b_{i}\right]}|X| \quad \text { (by (2.2)) } \\
& \leq \mathcal{O}(1)+\alpha^{-2} \beta C \sum_{i=m_{0}}^{\infty} \int_{\left[b_{i-1}<|X| \leq b_{i}\right]}|X| a(\log |X|)<\infty .
\end{aligned}
$$


Note that

$$
\begin{aligned}
E\left[\sum_{n=1}^{\infty} E\left(W_{n}^{2} \mid \mathcal{F}_{n-1}\right)\right] & \leq E\left[\sum_{n=1}^{\infty} E\left(\frac{Y_{n}^{2}}{b_{n}^{2}} \mid \mathcal{F}_{n-1}\right)\right] \\
& =\sum_{n=1}^{\infty} E \frac{Y_{n}^{2}}{b_{n}^{2}}<\infty,
\end{aligned}
$$

which implies that $\sum_{n=1}^{\infty} E\left(W_{n}^{2} \mid \mathcal{F}_{n-1}\right)<\infty$ a.c. Hence, by Lemma 1 , we have $\sum_{n=1}^{\infty} W_{n}$ a.c. convergence.

Theorem 1 follows from (2.5) and (2.7).

Theorem 1 also includes some particular cases of means, we can establish the following.

Corollary 1 Let $\left\{X, X_{n}, n \in \mathbb{N}\right\}$ be a sequence of random variables with $\left\{X_{n}\right\} \prec X$. If for some $\varepsilon>0, E \frac{|X|}{\log |X| \mathbf{1}_{[|X|>\varepsilon]}}<\infty$, then

$$
\lim _{n} \frac{1}{\log n} \sum_{k=1}^{n}\left[\frac{X_{k}-E\left(X_{k} \mathbf{1}_{\left[\left|X_{k}\right| \leq k \log k\right]} \mid \mathcal{F}_{k-1}\right)}{k}\right]=0, \quad \text { a.c. }
$$

Corollary 2 Let $\left\{X, X_{n}, n \in \mathbb{N}\right\}$ be a sequence of random variables with $\left\{X_{n}\right\} \prec X$ and for some $k \geq 2$,

$$
a_{n}=\left[n(\log n) \cdots\left(\log _{k-1} n\right)\right]^{-1},
$$

where $\log _{1} n=\log n, \log _{k} n=\log \left(\log _{k-1} n\right), k \geq 2$, iffor all large $C>0$,

$$
E \frac{|X| \mathbf{1}_{[|X|>C]}}{(\log |X|) \cdots\left(\log _{k}|X|\right)}<\infty
$$

then

$$
\lim _{n} \frac{1}{A_{n}} \sum_{k=1}^{n} a_{k}\left[X_{k}-E\left(X_{k} \mathbf{1}_{\left[\left|X_{k}\right| \leq b_{k}\right]} \mid \mathcal{F}_{k-1}\right)\right]=0, \quad \text { a.c. }
$$

Corollary 3 Let $\left\{X, X_{n}, n \in \mathbb{N}\right\}$ be a sequence of random variables with $\left\{X_{n}\right\} \prec X$. Further, let $\mathcal{F}_{n}=\sigma\left(X_{1}, \ldots, X_{n}\right)$ and $\mathcal{F}_{-n}=\{\phi, \Omega\}, n \geq 0$. If $E|X| a\left(\log ^{+}|X|\right)<\infty$, then for any $m \geq 1$,

$$
\lim _{n} \frac{1}{A_{n}} \sum_{k=1}^{n} a_{k}\left[X_{k}-E\left(X_{k} \mathbf{1}_{\left[\left|X_{k}\right| \leq b_{k}\right]} \mid \mathcal{F}_{k-m}\right)\right]=0, \quad \text { a.c. }
$$

Proof Since $\left\{X_{n m+l}, \mathcal{F}_{n m+l}, n \geq 1\right\}$ is an adapted stochastic sequence and $\left\{X_{n m+l}\right\} \prec X$, by Theorem 1 , we have for $l=0,1, \ldots, m-1$ that

$$
\sum_{n=1}^{\infty} \frac{X_{n m+l}-E\left(X_{n m+l} \mathbf{1}_{\left[\left|X_{n m+l}\right| \leq b_{n m+l}\right]} \mid \mathcal{F}_{(n-1) m+l}\right)}{b_{n m+l}} \quad \text { converges a.c. }
$$


Therefore, we have

$$
\begin{aligned}
& \sum_{n=m}^{\infty} \frac{X_{n}-E\left(X_{n} \mathbf{1}_{\left[\left|X_{n}\right| \leq b_{n}\right]} \mid \mathcal{F}_{n-m}\right)}{b_{n}} \\
& \quad=\sum_{l=0}^{m-1} \sum_{n=1}^{\infty} \frac{X_{n m+l}-E\left(X_{n m+l} \mathbf{1}_{\left[\left|X_{n m+l}\right| \leq b_{n m+l}\right]} \mid \mathcal{F}_{(n-1) m+l}\right)}{b_{n m+l}} \quad \text { converges a.c. }
\end{aligned}
$$

Corollary 4 Let $\left\{X_{n}, n \in \mathbb{N}\right\}$ be a sequence of m-dependent random variables. Further, let $\mathcal{F}_{n}=\sigma\left(X_{1}, \ldots, X_{n}\right)$ and $\mathcal{F}_{-n}=\{\phi, \Omega\}, n \geq 0$. If there exists a random variable $X$ such that $\left\{X_{n}\right\} \prec X$ and $E|X| a\left(\log ^{+}|X|\right)<\infty$, then

$$
\lim _{n} \frac{1}{A_{n}} \sum_{k=1}^{n} a_{k}\left[X_{k}-E\left(X_{k} \mathbf{1}_{\left[\left|X_{k}\right| \leq b_{k}\right]}\right)\right]=0, \quad \text { a.c. }
$$

Proof Note that $\left\{X_{n}, n \in \mathbb{N}\right\}$ is a sequence of $\mathrm{m}$-dependent random variables, then $E\left(X_{n} \mid \mathcal{F}_{n-m}\right)=E X_{n}$, Corollary 4 follows directly from Corollary 3.

Definition 2 (Stout, 1974) Let $\left\{X_{n}, n \in \mathbb{N}\right\}$ be a sequence of random variables, and let $\mathcal{F}_{n}^{m}=$ $\sigma\left(X_{n}, \ldots, X_{m}\right)$. We say that the sequence $\left\{X_{n}, n \in \mathbb{N}\right\}$ is $*$-mixing if there exists a positive integer $M$ and a non-decreasing function $\varphi(n)$ defined on integers $n \geq M$ with $\lim _{n} \varphi(n)=$ 0 such that for $n>M, A \in \mathcal{F}_{0}^{m}$ and $B \in \mathcal{F}_{m+n}^{\infty}$, the relation

$$
|\mathbb{P}(A \cap B)-\mathbb{P}(A) P(B)| \leq \varphi(n) \mathbb{P}(A) \mathbb{P}(B)
$$

holds for any integer $m \geq 1$.

It has been proved ( $c f .[4])$ that the *-mixing condition is equivalent to the condition

$$
\left|\mathbb{P}\left(B \mid \mathcal{F}_{0}^{m}\right)-\mathbb{P}(B)\right| \leq \varphi(n) \mathbb{P}(B), \quad \text { a.c. }
$$

for $B \in \mathcal{F}_{m+n}^{\infty}$ and $m \geq 1$ implies

$$
\left|E\left(X_{n+m} \mid \mathcal{F}_{0}^{m}\right)-E X_{n+m}\right| \leq \varphi(n) E\left|X_{n+m}\right|, \quad \text { a.c. }
$$

Theorem 2 Let $\left\{X, X_{n}, n \in \mathbb{N}\right\}$ be a sequence of ${ }^{*}$-mixing random variables with $\left\{X_{n}\right\} \prec X$. Further, let $\mathcal{F}_{n}=\sigma\left(X_{1}, \ldots, X_{n}\right)$ and $\mathcal{F}_{-n}=\{\phi, \Omega\}, n \geq 0$. If $\max \left\{E|X|, E|X| a\left(\log ^{+}|X|\right)\right\}<\infty$, then

$$
\lim _{n} \frac{1}{A_{n}} \sum_{k=1}^{n} a_{k}\left[X_{k}-E X_{k} \mathbf{1}_{\left[\left|X_{k}\right| \leq b_{k}\right]}\right]=0, \quad \text { a.c. }
$$

Proof By Corollary 3, we have, for each $m \geq 1$,

$$
\lim _{n} \frac{1}{A_{n}} \sum_{k=1}^{n} a_{k}\left[X_{k}-E\left(X_{k} \mathbf{1}_{\left[\left|X_{k}\right| \leq b_{k}\right]} \mid \mathcal{F}_{k-m}\right)\right]=0, \quad \text { a.c. }
$$


Since $\left\{X_{n}, n \in \mathbb{N}\right\}$ is $*$-mixing, by (2.8) and (2.9), we obtain

$$
\begin{aligned}
& \left|\frac{1}{A_{n}} \sum_{k=1}^{n} a_{k}\left[X_{k}-E X_{k} \mathbf{1}_{\left[\left|X_{k}\right| \leq b_{k}\right]}\right]\right| \\
& \leq\left|\frac{1}{A_{n}} \sum_{k=1}^{n} a_{k}\left[X_{k}-E\left(X_{k} \mathbf{1}_{\left[\left|X_{k}\right| \leq b_{k}\right]} \mid \mathcal{F}_{k-m}\right)\right]\right| \\
& \quad+\frac{1}{A_{n}} \sum_{k=1}^{n} a_{k}\left|\left[E\left(X_{k} \mathbf{1}_{\left[\left|X_{k}\right| \leq b_{k}\right]} \mid \mathcal{F}_{k-m}\right)-E X_{k} \mathbf{1}_{\left[\left|X_{k}\right| \leq b_{k}\right]}\right]\right| \\
& \leq\left|\frac{1}{A_{n}} \sum_{k=1}^{n} a_{k}\left[X_{k}-E\left(X_{k} \mathbf{1}_{\left[\left|X_{k}\right| \leq b_{k}\right]} \mid \mathcal{F}_{k-m}\right)\right]\right|+\frac{\varphi(m)}{A_{n}} \sum_{k=1}^{n} a_{k} E\left|X_{k}\right| \mathbf{1}_{\left[\left|X_{k}\right| \leq b_{k}\right]} \\
& \left.\leq\left|\frac{1}{A_{n}} \sum_{k=1}^{n} a_{k}\left[X_{k}-E\left(X_{k} \mathbf{1}_{\left[\left|X_{k}\right| \leq b_{k}\right]} \mid \mathcal{F}_{k-m}\right)\right]\right|+\varphi(m) E|X| \rightarrow 0 \quad \text { a.c. (as } n \rightarrow \infty\right) .
\end{aligned}
$$

Thus, using the Kroneker lemma, Theorem 2 follows.

\section{Competing interests}

The authors declare that they have no competing interests.

\section{Authors' contributions}

WZ and FA carried out the design of the study and performed the analysis, WZ drafted the manuscript. All authors read and approved the final manuscript.

\section{Acknowledgements}

Foundation item: The National Nature Science Foundation of China (No. 11071104), Foundation of Anhui Educational Committee (KJ2012B117) and Graduate Innovation Fund of AnHui University of Technology (D2011025).

Received: 28 August 2012 Accepted: 3 January 2013 Published: 18 January 2013

\section{References}

1. Chow, YS, Teicher, H: Almost certain summability of independent, identically distributed random variables. Ann. Math. Stat. 42(1), 401-404 (1971)

2. Adler, Y, Rosasky, A: Some general strong laws for weighted sums of stochastically dominated random variables. Stoch. Anal. Appl. 5(1), 1-16 (1987)

3. Chow, YS, Teicher, H: Probability Theory. Springer, New York (1978)

4. Stout, WF: Almost Sure Convergence. Academic Press, San Diego (1974)

doi:10.1186/1029-242X-2013-25

Cite this article as: Fan and Wang: On almost sure limiting behavior of a dependent random sequence. Journal of Inequalities and Applications 2013 2013:25.

\section{Submit your manuscript to a SpringerOpen ${ }^{\circ}$ journal and benefit from:}

- Convenient online submission

Rigorous peer review

- Immediate publication on acceptance

- Open access: articles freely available online

- High visibility within the field

- Retaining the copyright to your article 